\title{
Coupling the dynamics and the molecular chemistry in the Galactic center ${ }^{\star}$
}

\author{
N. J. Rodriguez-Fernandez ${ }^{1,2}$, F. Combes ${ }^{3}$, J. Martin-Pintado ${ }^{4}$, T. L. Wilson ${ }^{5}$, and A. Apponi ${ }^{6}$ \\ 1 Observatoire de Bordeaux, L3AB (UMR 5804)/OASU, CNRS/Université Bordeaux 1, BP 89, 2 rue de l'Observatoire, \\ 33270 Floirac, France \\ e-mail: nemesio.rodriguez@obs.u-bordeaux1.fr \\ 2 Université Denis Diderot (Paris VII) \& Observatoire de Paris, 61 Av de l'Observatoire, 75014 Paris, France \\ 3 LERMA, Observatoire de Paris, 61 Av de l'Observatoire, 75014 Paris, France \\ 4 DAMIR, IEM, CSIC, Serrano 121, Madrid, Spain \\ 5 ESO, Karl-Schwarzschild-Str. 2, 85748 Garching bei München, Germany \\ ${ }^{6}$ Steward Observatory, University of Arizona, Tucson, AZ 85721, USA
}

Received 5 January 2006 / Accepted 10 April 2006

ABSTRACT

\begin{abstract}
Most of the Galactic center (GC) gas moves in nearly circular orbits in a nuclear ring (hereafter the Galactic center ring, GCR). This is the case of cloud complexes such as Sgr A or Sgr B, where the gas is dense, warm and exhibits a rich molecular chemistry. The origin of these properties is thought to be shocks, in particular due to the large scale dynamics of the Galaxy. In addition, there are gas clouds moving in highly non-circular orbits known from observations of low density tracers such as $\mathrm{CO}(1-0)$. The physical conditions of the clouds moving with non-circular velocities are not well known. We have studied the physical conditions of the gas in non-circular orbits to better understand the origin of the unusual physical conditions of the GC molecular gas and the possible effect of the large scale dynamics on these physical conditions. Using published $\mathrm{CO}(1-0)$ data, we have selected a set of clouds belonging to all the kinematical components seen in the longitude-velocity diagram of the GC. We have carried out a survey of dense gas in all the components using the $J=2-1$ lines of CS and SiO as tracers of high density gas and shock chemistry. We have detected CS and $\mathrm{SiO}$ emission in all the kinematical components. The gas density and the $\mathrm{SiO}$ abundance of the clouds in non-circular orbits are similar to those in the GCR. Therefore, in all the kinematical components there are dense clouds that can withstand the tidal shear. However, there is no evidence of star formation outside the GCR. The high relative velocity and shear expected in the dust lanes along the bar major axis could inhibit the star formation process, as observed in other galaxies. The high SiO abundances derived in the non-circular velocity clouds are likely due to the large-scale shocks that created the dust lanes.
\end{abstract}

Key words. ISM: kinematics and dynamics - ISM: molecules - galaxies: ISM - radio lines : ISM

\section{Introduction}

The longitude-latitude maps of the Galactic center (GC) show several major cloud complexes: the $l=1.3^{\circ}$-complex (a huge molecular complex located at longitudes between $l=1.2^{\circ}$ and $\left.l=1.6^{\circ}\right)$, the Sgr D complex at $l=1^{\circ}$, the Sgr B complex near $l=0.7^{\circ}$, the Sgr A cloud near $l=0^{\circ}$ and the Sgr C cloud near $l=-0.5^{\circ}$ (see for instance, Scoville 1972; Liszt \& Burton 1978; Bania 1977; Bally et al. 1987; Dahmen et al. 1997; Oka et al. 1998a,b; Bitran et al. 1997). In the longitude-velocity diagram (hereafter $l v$-diagram, see Fig. 1), the Sgr A... D complexes are located approximately along a line passing through the origin as expected for gas moving along an almost circular ring. Therefore, in a face-on view of the Galactic center these complexes would be located in a circular ring (Fig. 2). Sofue (1995) proposed that this ring is composed of two arms (Arm I and Arm II). Hereafter, we refer to this structure as the Galactic center ring (GCR). However, the GCR clouds and the $l=1.3^{\circ}$-complex are not the only cloud components in the GC. The $l v$-diagram also exhibits many structures corresponding to clouds moving in non-circular trajectories like the Clump 2, the Connecting Arm, or the structures that we have labeled as

* Figure 3 and Table 2 are only available in electronic form at http://www . edpsciences.org
J, K, L, M, N, O and P (Fig. 1). The easiest explanation for the non-circular trajectories is the gas response to a bar potential (Binney et al. 1991; Fux 1999).

In the inner region of a barred galaxy there are two main families of closed orbits in the reference frame of the bar (Contopoulos \& Papayannopoulos 1980): the $x_{1}$ orbits, which are elongated along the bar major axis and the $x_{2}$ orbits, which are elongated along the minor axis of the bar, when there exists inner Linblad resonances (ILR). Numerical simulations of the gas dynamics (Athanassoula 1992; Fux 1999) show that the gas in the center tends to follow nearly circular $x_{2}$ orbits creating a nuclear ring (the GCR). At larger radii, beyond the ILRs, the gas tends to follow $x_{1}$ orbits along the bar. In fact, the gas clouds are subject to collisions, and do not follow strictly these orbits, but are frequently kicked off into neighboring orbits, in general with lower energy. In particular, at the transition region between the $x_{1}$ and $x_{2}$ flows, collisions are favored, and there might be a scatter, or "spray" of gas clouds moving along the $x_{1}$ flow. The sprayed gas will collide with the material on the opposite side of the bar major axis giving rise to shocks in the molecular cloud component and to the characteristic dust lanes that are observed near the leading edges of the bars in external galaxies. Losing energy and angular momentum, the gas of the dust lanes will progressively spiral down to the nuclear ring and it will fall in after only a few rotations (see Fig. 20 of Fux 1999). 


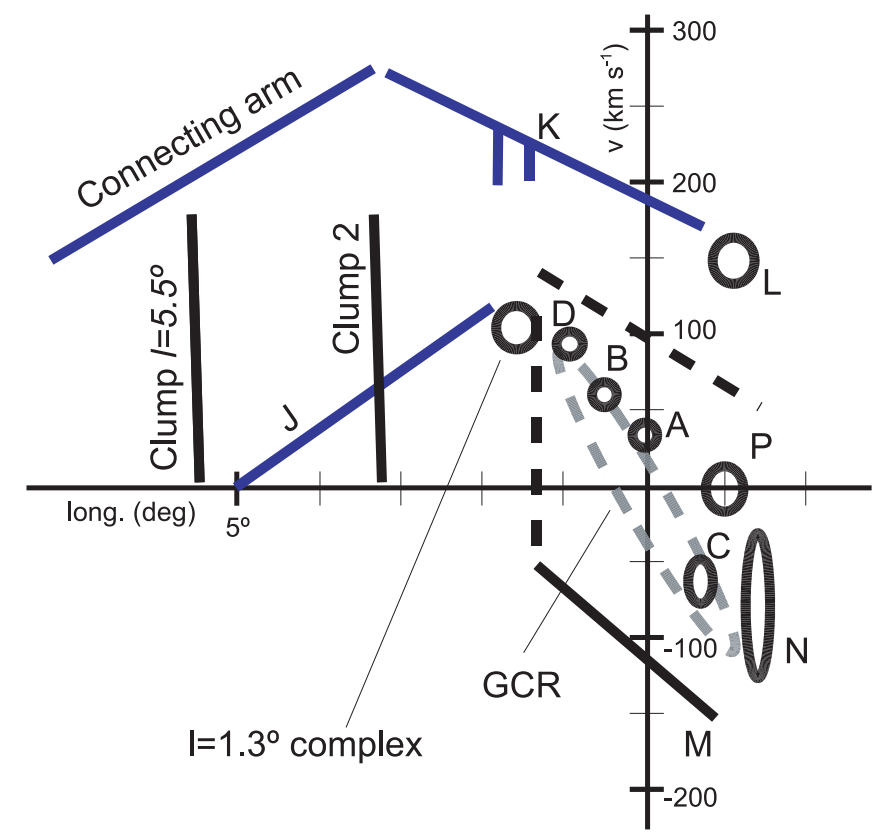

Fig. 1. Schema illustrating the main kinematical structures in the longitude-velocity diagram of the inner degrees of the Galaxy. The labels A to D stand for Sgr A to Sgr D complexes. See the text for an explanation of the other labels.

In the GC, Fux (1999) has identified the Connecting Arm as the near-side dust lane. The structure $\mathrm{K}$, which seems to be linked to the Connecting Arm at $l \sim 3^{\circ}$ (Fig. 1), represents gas clouds that move in the decelerating section of elongated orbits, i.e, gas that does not fall in the nuclear ring at the first passage around the $x_{1}-x_{2}$ interaction region (see Fig. 2). The structure $\mathbf{J}$ has nearly the same inclination as that of the Connecting Arm (Fig. 1), and it could be a kind of small dust lane, but in contrast with the Connecting Arm and the structure $\mathrm{K}$, the gas in the structure $\mathrm{J}$ will easily interact with the material of the nuclear disk in the area of the $l=1.3^{\circ}$-complex (see Fig. 2). Thus, the $l=1.3^{\circ}$-complex would be the contact point of the dust lane and the GCR as proposed by Hüttemeister et al. (1998) and Fux (1999). The clump at $l=5^{\circ}$ and the Clump 2 are gas clouds that are about to enter the dust lane shock. The $l v$-diagram at $l<0$ is more difficult to interpret. The $l v$-diagram is not expected to be symmetric. Due to perspective effects the far side dust lane is elongated along the line of sight and thus it is a vertical structure in the $l v$-diagram close to the extremity of the nuclear ring (see Fig. 23 of Fux 1999). The complexes L, N, and P could indeed belong to the far-side dust lane shock. The structure $M$ would be part of the gas spray due to the interaction of the far-side dust lane and the GCR.

The physical conditions of the neutral gas in the GC have been studied by observing high density tracers like CS (Bally et al. 1988; Tsuboi et al. 1999) or HCN (Jackson et al. 1996) and by multi-level studies of $\mathrm{NH}_{3}$ (Hüttemeister et al. 1998), $\mathrm{H}_{2}$ (Rodríguez-Fernández et al. 2001, 2004) or CO (Sawada et al. 2001; Martin et al. 2004). These works, among others, have shown that the clouds in the GCR and the $l=1.3^{\circ}$-complex are dense $\left(10^{4} \mathrm{~cm}^{-3}\right)$ and warm (up to $\sim 150 \mathrm{~K}$ ). In addition, the GCR clouds present widespread emission and high abundances of molecules like $\mathrm{SiO}, \mathrm{SO}, \mathrm{CH}_{3} \mathrm{OH}$, or $\mathrm{C}_{2} \mathrm{H}_{5} \mathrm{OH}$ (Martín-Pintado et al. 1997, 2001; Lis et al. 2001; Hüttemeister et al. 1998), which are considered good shock tracers. Indeed, shocks are commonly invoked to explain the molecular chemistry and the high temperatures of the neutral

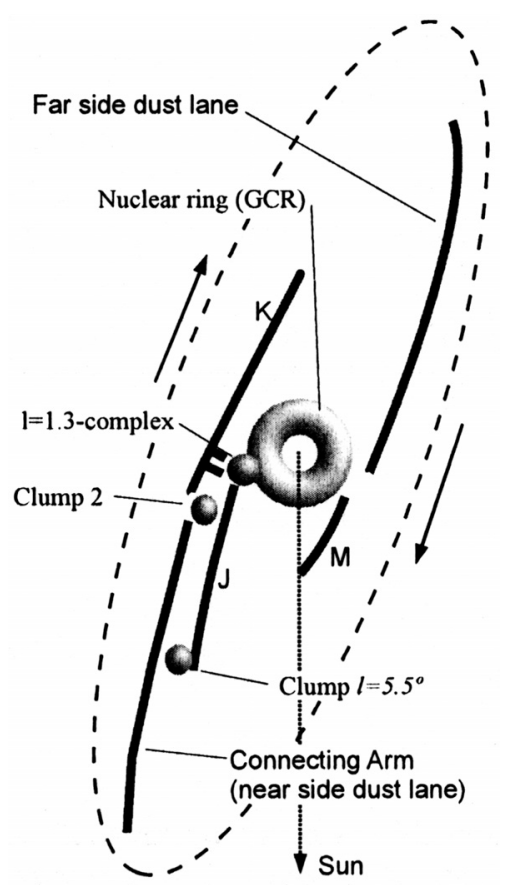

Fig. 2. Schema of a plausible face on view of the Galactic center.

gas in the GCR, the $l=1.3^{\circ}$-complex and the Clump 2 (Martín-Pintado et al. 1997, 2001; Hüttemeister et al. 1993, 1998; Lis et al. 2001; Rodríguez-Fernández et al. 2004). The origin of the shocks could be related to Wolf-Rayet winds (Martín-Pintado et al. 1999) or to cloud-cloud collisions induced by the large scale dynamics of the Galaxy (Wilson et al. 1982; Hüttemeister et al. 1998; Martín-Pintado et al. 2001; Sato et al. 2000; Rodríguez-Fernández et al. 2000, 2004).

In contrast to the GCR and the $l=1.3^{\circ}$-complex, little is known about the physical conditions of the GC clouds with noncircular velocities. Up to now, the only large scale maps that have been used to study the physical conditions of the ensemble of the GC molecular gas are those of Oka et al. (1998a) and Sawada et al. (2001). They have mapped the $\mathrm{CO}(2-1)$ and $\mathrm{CO}(1-0)$ lines and found a rather constant line ratio $(\sim 1)$ in the GC clouds (including Clump 2). A few clouds of the upper-right corner of the $l v$-diagram have been observed in $\mathrm{SiO}$ and $\mathrm{NH}_{3}$ by Hüttemeister et al. $(1993,1998)$ and in $\mathrm{H}_{2}$ and fine structure lines by Rodríguez-Fernández et al. (2004). Their densities, temperatures and excitation mechanisms seem to be similar to the GCR clouds.

Thus, in contrast to the GCR cloud complexes, the physical conditions of the gas with non-circular velocities that constitute the characteristic shape of the $l v$-diagram are not well-known. However, the study of the properties of all these kinematical components can give us important hints on the origin of the outstanding physical conditions of the GC clouds and on the possible links between these physical conditions and the global gas dynamics. This is the purpose of this paper. We have selected a sample of clouds belonging to all the kinematic components seen in the GC $l v$-diagram and we have undertaken a survey of dense gas using $\mathrm{CS}$ and $\mathrm{SiO}$ as tracers. Due to their high dipole moments, both $\mathrm{CS}$ and $\mathrm{SiO}$ are high density tracers $\left(\geq 10^{4} \mathrm{~cm}^{-3}\right)$. In addition, the $\mathrm{SiO}$ molecule traces dense gas affected by shocks (Martin-Pintado et al. 1992). Since the CS and SiO molecules are quite similar and they have similar energy level distributions, the $\mathrm{SiO} / \mathrm{CS}$ line ratio is expected to depend mainly on the relative abundance of the two molecules. 
Table 1. Summary of the ${ }^{13} \mathrm{CO}(2-1)$ and $(1-0)$ observations. Intensity of the ${ }^{13} \mathrm{CO}(1-0)$ line $\left(T_{\mathrm{mb}}\right)$ and ${ }^{13} \mathrm{CO}(2-1) /(1-0)$ intensity ratio calculated from the $T_{\mathrm{mb}}$ temperatures without any correction for the different beam sizes. When the ${ }^{13} \mathrm{CO}(2-1)$ line has not been detected we have used $3 \sigma$ upper limits to calculate the $2-1 / 1-0$ ratio. The $\mathrm{LVG}$ results for $T_{\mathrm{K}}=20 \mathrm{~K}$ are also displayed. For the sources where the ${ }^{13} \mathrm{CO}(2-1) /(1-0)$ ratio could not be determined, we have assumed a $\mathrm{H}_{2}$ density of $n=10^{3-4.5} \mathrm{~cm}^{-3}$. The $\mathrm{SiO}$ column density, $N(\mathrm{SiO})$, has been calculated for a $\mathrm{H}_{2}$ density of $10^{4.5} \mathrm{~cm}^{-3}$. The $\mathrm{SiO}$ abundance relative to $\mathrm{H}_{2}, X(\mathrm{SiO})$, has been calculated using a ${ }^{13} \mathrm{CO}$ abundance of $5 \times 10^{-6}$ (see text).

\begin{tabular}{|c|c|c|c|c|c|c|c|c|c|}
\hline $\begin{array}{l}l \\
\operatorname{deg}\end{array}$ & $\begin{array}{l}b \\
\text { deg }\end{array}$ & $\begin{array}{l}v \\
\mathrm{~km} \mathrm{~s}^{-1}\end{array}$ & Feature & $\begin{array}{l}{ }^{13} \mathrm{CO}(1-0) \\
T_{\mathrm{mb}}(\mathrm{K})\end{array}$ & $2-1 / 1-0$ & $\begin{array}{l}\log (n(\mathrm{H} 2)) \\
\log \left(\mathrm{cm}^{-3}\right)\end{array}$ & $\begin{array}{l}N\left({ }^{13} \mathrm{CO}\right) \\
10^{16} \mathrm{~cm}^{-2}\end{array}$ & $\begin{array}{l}N(\mathrm{SiO}) \\
10^{13} \mathrm{~cm}^{-2}\end{array}$ & $\begin{array}{l}X(\mathrm{SiO}) \\
10^{-9}\end{array}$ \\
\hline-0.5030 & -0.0500 & 144.5 & $\mathrm{~L}$ & $0.8 \pm 0.2$ & $0.7 \pm 0.4$ & $3.0-4.0$ & 1.1 & $<1.1$ & $<5$ \\
\hline-0.5900 & -0.0500 & 152.8 & $\mathrm{~L}$ & $0.5 \pm 0.2$ & $<1.7$ & $<4.3$ & 0.38 & $<0.8$ & $<9.9$ \\
\hline-0.6880 & -0.0500 & 142.5 & $\mathrm{~L}$ & $1.0 \pm 0.7$ & $0.7 \pm 0.5$ & $3 .-3.5$ & 0.6 & 1.0 & 7.9 \\
\hline 0.9452 & -0.0500 & 86.2 & $\mathrm{~L}$ & $4.5 \pm 0.3$ & $1.0 \pm 0.1$ & $3.4-3.6$ & 12 & 7.3 & 3.0 \\
\hline 0.1500 & -0.0500 & 154.7 & $\mathrm{~K}$ & $1.2 \pm 0.1$ & $0.6 \pm 0.1$ & $3-3.5$ & 0.45 & $<0.3$ & $<3.1$ \\
\hline 1.3100 & -0.1300 & 204.3 & $\mathrm{~K}$ & $1.1 \pm 0.3$ & $\ldots$ & $\ldots$ & $2-4$ & $<0.2$ & $<0.33$ \\
\hline 0.3899 & -0.1500 & 195.8 & $\mathrm{~K}$ & $0.8 \pm 0.3$ & $\ldots$ & $\ldots$ & 0.8 & $<2.5$ & $<15$ \\
\hline 1.4027 & -0.1500 & 214.3 & $\mathrm{~K}$ & $1.0 \pm 0.1$ & $0.6 \pm 0.1$ & $3 .-3.4$ & 2 & 0.4 & 0.88 \\
\hline 1.5220 & -0.1500 & 219.5 & $\mathrm{~K}$ & $1.6 \pm 0.3$ & $\ldots$ & $\ldots$ & 2.5 & 0.2 & 0.4 \\
\hline 0.3899 & -0.1500 & -92.2 & M & $0.5 \pm 0.3$ & $1.3 \pm 0.8$ & $3.5-4.3$ & $0.2-1$ & 0.3 & $1.3-6.8$ \\
\hline 0.3899 & -0.1500 & -71.5 & M & $0.3 \pm 0.2$ & $<2.3$ & $<4.3$ & $>0.6$ & 0.6 & $<5$ \\
\hline 0.9452 & -0.0500 & -81.0 & M & $1.0 \pm 0.3$ & $1.0 \pm 0.4$ & $3 .-3.8$ & 1.1 & 0.7 & 3.2 \\
\hline 0.9452 & -0.0500 & -65.9 & M & $1.6 \pm 0.3$ & $0.7 \pm 0.2$ & $3 .-3.5$ & 2.8 & 0.4 & 0.63 \\
\hline-0.6880 & -0.0500 & -41.7 & $\mathrm{P}$ & $0.9 \pm 0.4$ & $1.5 \pm 0.7$ & $3.5-5$ & 1.7 & 2.7 & 7.9 \\
\hline-0.6880 & -0.0500 & -17.5 & $\mathrm{P}$ & $1.0 \pm 0.4$ & $2.2 \pm 0.9$ & $3.5-5$ & 1.0 & 3.2 & 16 \\
\hline-0.6880 & -0.0500 & 2.3 & $\mathrm{P}$ & $2.0 \pm 0.4$ & $1.5 \pm 0.3$ & $3.5-5$ & 1.0 & 5.0 & 25 \\
\hline-0.5900 & -0.0500 & -41.3 & $\mathrm{P}$ & $1.6 \pm 0.2$ & $0.9 \pm 0.2$ & $3.5-4$ & 2.2 & $<1.4$ & $<3.2$ \\
\hline 1.3100 & -0.1300 & -13.8 & 1.3 & $2.0 \pm 0.3$ & $0.8 \pm 0.2$ & $3.5-4.3$ & 5.7 & 0.2 & 0.16 \\
\hline 1.3100 & -0.1300 & 33.7 & 1.3 & $1.9 \pm 0.3$ & $1.0 \pm 0.2$ & $3.5-4.3$ & 5.7 & 9.5 & 8.3 \\
\hline 1.3100 & -0.1300 & 85.0 & 1.3 & $2.4 \pm 0.3$ & $1.1 \pm 0.2$ & $3.5-4.3$ & 7.5 & 5.3 & 3.5 \\
\hline 1.3100 & -0.1300 & 135.0 & 1.3 & $0.5 \pm 0.3$ & $<1.7$ & $<10^{4}$ & 0.4 & $<0.1$ & $<1.3$ \\
\hline 1.4027 & -0.1500 & -41.8 & 1.3 & $1.1 \pm 0.1$ & $0.6 \pm 0.1$ & 3. & 0.6 & $<0.1$ & $<1.0$ \\
\hline 1.4027 & -0.1500 & -22.9 & 1.3 & $1.5 \pm 0.3$ & $0.7 \pm 0.2$ & $3-3.5$ & 1.9 & 0.5 & 1.2 \\
\hline 1.4027 & -0.1500 & 29.7 & 1.3 & $1.5 \pm 0.2$ & $0.9 \pm 0.2$ & $3.3-3.5$ & $2.2-3.5$ & 1.4 & 2.5 \\
\hline 1.4027 & -0.1500 & 72.3 & 1.3 & $1.6 \pm 0.2$ & $0.9 \pm 0.1$ & $3.3-3.5$ & 6.6 & 2.3 & 1.7 \\
\hline 1.4027 & -0.1500 & 123.7 & 1.3 & $0.6 \pm 0.0$ & $1.3 \pm 0.1$ & $3.5-4.0$ & 0.85 & 0.5 & 3 \\
\hline 1.5220 & -0.1500 & -23.4 & GCR & $1.1 \pm 0.8$ & $0.8 \pm 0.6$ & $3.2-4$ & 2.3 & 0.5 & 1.0 \\
\hline 1.5220 & -0.1500 & 56.0 & GCR & $1.1 \pm 0.8$ & $0.9 \pm 0.7$ & $<4.3$ & 3.4 & 0.9 & 1.3 \\
\hline-0.6880 & -0.0500 & -129.1 & GCR & $2.6 \pm 0.4$ & $1.2 \pm 0.2$ & $3.5-4.5$ & 4.5 & 1.1 & 1.2 \\
\hline-0.5900 & -0.0500 & 132.9 & GCR & $0.3 \pm 0.0$ & $<3.3$ & $\ldots$ & 16 & $<0.8$ & $<0.25$ \\
\hline-0.5900 & -0.0500 & -148.5 & GCR & $1.1 \pm 0.2$ & $1.0 \pm 0.3$ & $3.5-4.5$ & 2.5 & $<2.6$ & $<5.2$ \\
\hline-0.5900 & -0.0500 & -116.7 & GCR & $5.3 \pm 0.2$ & $1.0 \pm 0.1$ & $>3.5$ & 9.5 & 3.0 & 1.6 \\
\hline-0.5030 & -0.0500 & -147.4 & GCR & $1.1 \pm 0.2$ & $0.6 \pm 0.3$ & $2.8-3.5$ & 0.5 & $0.1-0.8$ & $1.3-8$ \\
\hline-0.5030 & -0.0500 & -53.5 & GCR & $1.0 \pm 0.2$ & $1.3 \pm 0.4$ & $3.5-4.5$ & 1.4 & $0.7-2.1$ & $2.5-7.5$ \\
\hline 0.1500 & -0.0500 & 25.5 & GCR & $4.0 \pm 0.4$ & $1.2 \pm 0.2$ & $>3.5$ & 6 & $<1$ & 0.79 \\
\hline 0.1500 & -0.0500 & 61.1 & GCR & $5.3 \pm 0.4$ & $1.4 \pm 0.1$ & $>3.5$ & 14 & 1.5 & 0.54 \\
\hline 0.8300 & -0.1800 & 31.3 & GCR & $5.0 \pm 0.5$ & $1.0 \pm 0.1$ & $>3.5$ & 16 & 6.4 & 2 \\
\hline 0.8300 & -0.1800 & 56.9 & GCR & $0.5 \pm 0.5$ & $1.3 \pm 1.5$ & $3.5-4.5$ & 1.1 & 8.5 & 30 \\
\hline 0.8300 & -0.1800 & 99.1 & GCR & $1.8 \pm 0.5$ & $1.0 \pm 0.3$ & $3.2-4.5$ & 1.4 & 1.4 & 5 \\
\hline 0.4100 & 0.0300 & -51.0 & GCR & $1.1 \pm 0.5$ & $1.2 \pm 0.6$ & $3.5-4.2$ & 1.9 & 2.5 & 6.6 \\
\hline 0.4100 & 0.0300 & -21.9 & GCR & $1.5 \pm 0.5$ & $0.8 \pm 0.3$ & $3 .-3.5$ & 3.3 & 1.1 & 1.7 \\
\hline 0.4100 & 0.0300 & 17.0 & GCR & $3.9 \pm 0.5$ & $1.0 \pm 0.2$ & $3.5-4.3$ & 6.8 & 12 & 8.8 \\
\hline 0.4100 & 0.0300 & 37.9 & GCR & $5.1 \pm 0.5$ & $1.1 \pm 0.1$ & $>3.5$ & 8.2 & $<1.3$ & $<0.79$ \\
\hline 0.1000 & -0.1200 & -2.0 & GCR & $0.8 \pm 0.0$ & $2.2 \pm 0.7$ & $>3.5$ & 1.1 & 0.9 & 4 \\
\hline 0.1000 & -0.1200 & 30.1 & GCR & $6.0 \pm 0.6$ & $1.0 \pm 0.2$ & $3.5-4.3$ & 25 & 8.2 & 1.64 \\
\hline 0.1000 & -0.1200 & 68.1 & GCR & $1.9 \pm 0.6$ & $1.4 \pm 0.6$ & $>3.5$ & 3.8 & 0.9 & 1.2 \\
\hline 0.3899 & -0.1500 & -3.5 & GCR & $1.8 \pm 0.2$ & $1.5 \pm 0.2$ & $>3.7$ & 1.9 & $<0.3$ & $<0.7$ \\
\hline
\end{tabular}

\section{Observations}

We have selected 61 positions in the longitude range $3.5^{\circ}>l>$ $-1.5^{\circ}$ using the Bally et al. (1987) $\mathrm{CO}(1-0)$ data cube. Due to the crowded velocity fields in the GC, with these 61 pointings we have been able to study 161 clouds (both GCR and non-GCR clouds). Table 2 presents the Galactic coordinates and velocities of the different clouds. For an easy presentation and discussion of the results we have grouped the clouds that occupy the same region in the $(l, b, v)$ space or that belong to one of the coherent kinematical structures discussed above.
We have observed 43 of the selected positions in the $J=$ 2-1 line of CS and $\mathrm{SiO}$ using the University of Arizona 12 meter antenna at Kitt Peak in February 2003. The half power beam width of the telescope is $68^{\prime \prime}$ and $77^{\prime \prime}$ at the frequencies of the $\mathrm{CS}$ and $\mathrm{SiO}$ lines, respectively. The temperatures, in the $T_{\mathrm{R}}^{*}$ scale, have been converted to main-beam temperatures $\left(T_{\mathrm{mb}}\right)$ using the expression $T_{\mathrm{mb}}=T_{\mathrm{R}}^{*} / \eta_{\mathrm{m}}^{*}$ with corrected main beam efficiencies $\eta_{\mathrm{m}}^{*}$ of 0.95 and 0.94 for the $\mathrm{SiO}$ and CS spectra, respectively. As backends, we used filter-banks with $1 \mathrm{MHz}$ channels, which provide a velocity resolution of 3.06 and $3.45 \mathrm{~km} \mathrm{~s}^{-1}$ for the $\mathrm{CS}$ and $\mathrm{SiO}$ lines, respectively. The rms noise in $1 \mathrm{MHz}$ 

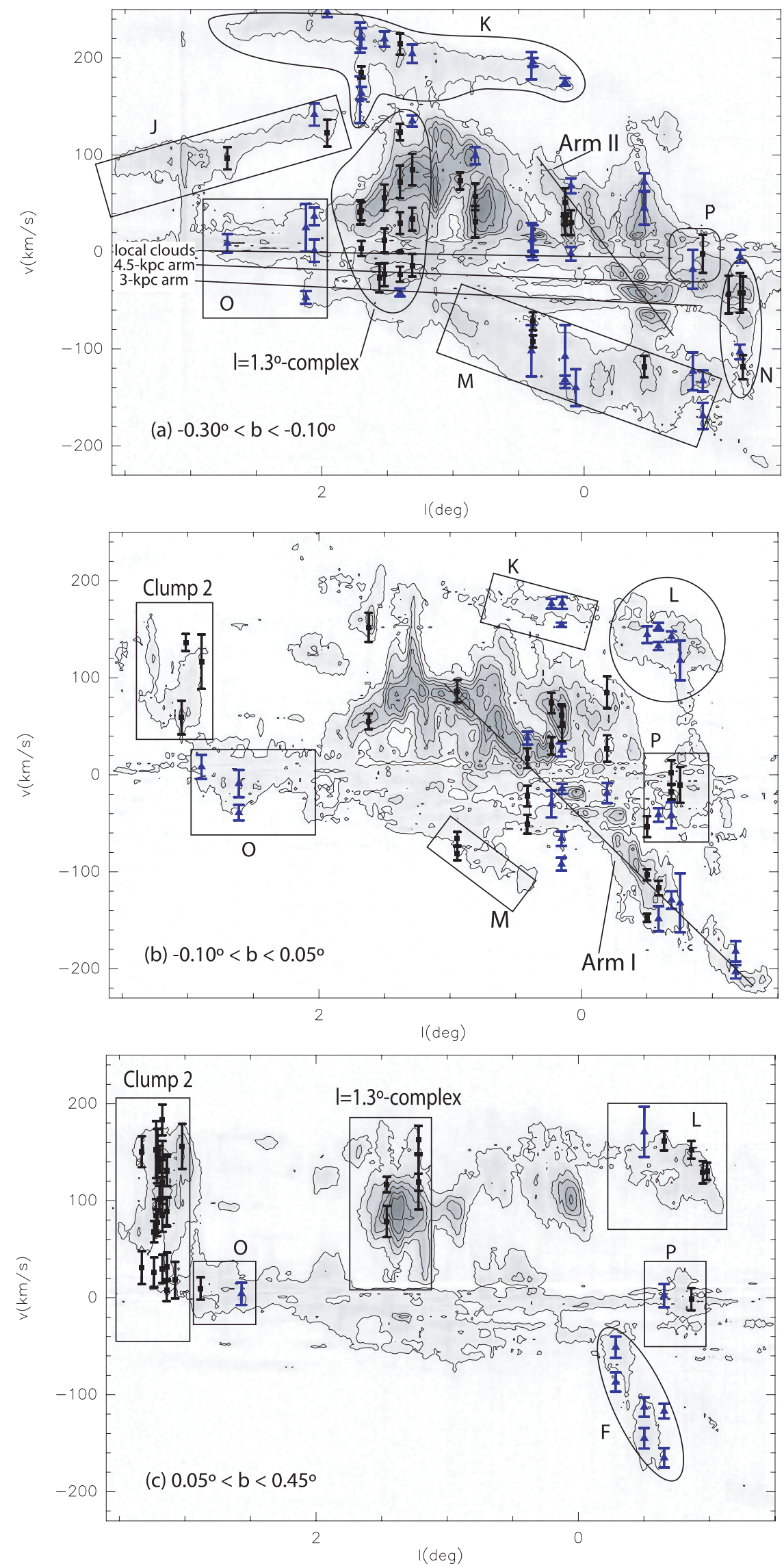

Fig. 4. In grey-levels and contours we show the longitude-velocity diagram obtained with the CO(1-0) data by Bally et al. (1987) at different latitude ranges. We also indicate the different kinematical features discussed in this paper (note that not all the features that are seen in different panels are labeled in all of them). Blue triangles and bars represent the peak velocity and the full width at half maximum (FWHM) of the sources detected in CS. Black points and bars show the peak velocity and FWHM of the sources that have been detected in CS and SiO. 
channels is in the range $0.04-0.11 \mathrm{~K}$ for the CS spectra and in the range $28-51 \mathrm{mK}$ for the $\mathrm{SiO}$ spectra.

The other 18 positions were observed with the IRAM $30 \mathrm{~m}$ telescope in July 2003. The two $100 \mathrm{GHz}$ receivers were used to simultaneously observe the two lines. The $30 \mathrm{~m}$ telescope beam size at the frequencies of the $\mathrm{CS}$ and $\mathrm{SiO}$ lines is 25.5" and $29^{\prime \prime}$ respectively. The temperatures, in the $T_{\mathrm{A}}^{*}$ scale, have been converted to main beam temperatures using the expression $T_{\mathrm{mb}}=\left(F_{\text {eff }} / B_{\text {eff }}\right) T_{\mathrm{A}}^{*}$ with a forward efficiency $\left(F_{\text {eff }}\right)$ of 0.95 and beam efficiencies $\left(B_{\text {eff }}\right)$ of 0.78 and 0.77 for the $\mathrm{SiO}$ and CS spectra, respectively. As backends, we also used filter-banks with $1 \mathrm{MHz}$ channels. The rms noise in $1 \mathrm{MHz}$ channels vary from source to source in the range $15-75 \mathrm{mK}$ for the CS spectra and in the range $11-57 \mathrm{mK}$ for the $\mathrm{SiO}$ spectra. The lowest rms are needed to obtain good detections of some clouds with non-circular velocities as those in the structure K. For comparison, the large scale CS(2-1) map of Bally et al. (1987) had a rms noise of $0.15 \mathrm{~K}$ in $1 \mathrm{MHz}$ filters.

We have also observed the ${ }^{13} \mathrm{CO}(2-1)$ and (1-0) lines towards 12 of the positions observed with the $30 \mathrm{~m}$ telescope (see Table 1). The IRAM $30 \mathrm{~m}$ beam size at the frequency of these lines is $11^{\prime \prime}$ and $22^{\prime \prime}$, respectively. The temperatures have been converted to $T_{\mathrm{mb}}$ using forward and beam efficiencies of 0.95 and 0.75 for the ${ }^{13} \mathrm{CO}(1-0)$ line and 0.91 and 0.55 for the ${ }^{13} \mathrm{CO}(2-1)$ line. All the observations were done in position switching mode. The emission free OFF positions have been carefully selected from the Oka et al. (1996) maps and our own spectra of different observational campaigns.

\section{Results}

Figure 3 shows the $\mathrm{CS}$ and $\mathrm{SiO}$ spectra obtained towards all the observed positions and Table 2 gives the velocity, widths and intensities of the CS lines as derived from Gaussian fits to the 161 lines detected. When the $\mathrm{SiO}$ lines are detected, the velocities and the linewidths are in agreement within errors with those of the CS lines and Table 2 shows the $\mathrm{SiO}$ to CS intensity ratio without any correction by the slightly different telescope beams. For the clouds where the $\mathrm{SiO}$ line has not been detected, Table 2 gives upper limits to the $\mathrm{SiO}(2-1) / \mathrm{CS}(2-1)$ line ratio as derived from the $3 \sigma$ limits to the $\mathrm{SiO}$ intensity. The results are also displayed graphically in Fig. 4. Blue triangles and bars represent the peak velocity and the full width at half maximum (FWHM) of the sources detected in CS. Black points and bars show the peak velocity and FWHM of the sources detected in CS and SiO.

We have detected CS emission in all the kinematical structures of the GC $l v$-diagram (Fig. 4, Table 2). The large linewidths measured (8-64 $\mathrm{km} \mathrm{s}^{-1}$ ) are typical of Galactic center clouds. The CS intensities range between the high values $(0.5-1.5 \mathrm{~K})$ measured for the sources in the $l=1.3^{\circ}$-complex, the Clump 2 and the GCR clouds to $0.1-0.3 \mathrm{~K}$ in the faint structures that constitute the upper and lower edges of the $l v$-diagram $(\mathrm{K}, \mathrm{M}, \mathrm{R})$, including the vertical appendix of the component $\mathrm{K}$ at $l \sim 1.7^{\circ}$, which can be the signature of mass transfer from the elongated orbits to the GCR (Fux 1999). The clouds in the right edge of the $l v$-diagram $(\mathrm{L}, \mathrm{N}, \mathrm{P})$ and those in the structure $\mathrm{J}$ exhibit intermediate CS intensities $(0.2-0.6 \mathrm{~K})$. The observations presented here demonstrate that there are dense CS-emitting clouds in all the kinematical components seen in the CO $l v$-diagram and not only in the GCR and the $l=1.3^{\circ}$-complex clouds detected by Bally et al. (1987).

We have also detected $\mathrm{SiO}(2-1)$ emission in at least a few clouds of all the kinematical components. The $\mathrm{SiO}(2-1)$ line has been detected in all the observed clouds of the $l=1.3^{\circ}$-complex and Clump 2. The $\mathrm{SiO} / \mathrm{CS}$ line ratio is in the range 0.3-0.6. The $\mathrm{SiO}$ line has also been detected in two clouds of the component $\mathrm{K}$ : one cloud near the $\mathrm{CO}$ maximum at $l \sim 1.4$ and one cloud in the vertical appendix at $l \sim 1.7^{\circ}$. The $\mathrm{SiO} / \mathrm{CS}$ ratio is 0.14 and 0.5 , respectively. $\mathrm{SiO}$ emission has also been detected in the lower edge (component $\mathrm{M}$ ) and the negative longitude extremity of the $l v$-diagram (components $\mathrm{P}$ and $\mathrm{N}$, including a few clouds with different velocities along the structure $\mathrm{N}$ ). The $\mathrm{SiO} / \mathrm{CS}$ ratios are in the range $0.2-0.5$. Similar line ratios have been measured towards several positions in the structures $\mathbf{J}$ and $\mathrm{L}$. In most of the clouds where the $\mathrm{SiO}$ line has not been detected the $\mathrm{CS}$ is indeed rather weak $(\sim 0.1 \mathrm{~K})$ and the upper limits to the $\mathrm{SiO} / \mathrm{CS}$ line ratio are not significant.

\section{Gas density and cloud stability}

To obtain further insight in the physical conditions in the different kinematical structures we have obtained ${ }^{13} \mathrm{CO}(1-0)$ and (2-1) data for some sources. Table 1 shows ${ }^{13} \mathrm{CO}(1-0)$ line intensities and ${ }^{13} \mathrm{CO}(2-1) /(1-0)$ line ratios without any correction by the different beams. The line ratio is $0.6-1.5$ for most of the sources (it would be a factor of two higher if the emission were extended and homogeneous). We have used a Large Velocity Gradient (LVG) code to determine the $\mathrm{H}_{2}$ density and the ${ }^{13} \mathrm{CO}$ column density for two values of the kinetic temperature $\left(T_{\mathrm{K}}\right)$ : 20 and $150 \mathrm{~K}$. Table 1 shows the results obtained for a kinetic temperature of $20 \mathrm{~K}$. The measured line ratios imply an $\mathrm{H}_{2}$ densitiy of $10^{3.5}-10^{4.5} \mathrm{~cm}^{-3}$ in the GCR clouds and $10^{3}-10^{3.5} \mathrm{~cm}^{-3}$ in the components $\mathrm{K}$ or $\mathrm{M}$. This is in perfect agreement with the results of Sawada et al. (2001) and Martin et al. (2004). Assuming a kinetic temperature of $150 \mathrm{~K}$ (Hüttemeister et al. 1993; Rodríguez-Fernández et al. 2000, 2001) the $\mathrm{H}_{2}$ density would be a factor of 3 lower.

In addition, the detection of the $\mathrm{CS}(2-1)$ and $\mathrm{SiO}(2-1)$ lines in all the velocity components imply the presence of high densities $\left(\geq 10^{4} \mathrm{~cm}^{-3}\right.$, Linke \& Goldsmith 1980) in the core of the clouds. Therefore, all the structures seen in the CO $l v$-diagram contain dense molecular gas. In particular, this is true for the gas moving in elongated orbits related to the dust lanes (like the components $\mathrm{J}$ and $\mathrm{K}$ ) or about to enter the dust lanes shocks (Clump 2, see also Stark \& Bania 1986). This result is in agreement with what is found in external galaxies, where the density contrast between the dust lanes and the nuclear ring is low (see for instance the results on NGC 5383 by Sheth et al. 2000).

The density of the clouds is directly related to the problem of cloud stability, which is of crucial interest to understand the star formation process. Due to the strong tidal forces in the Galactic center, the clouds must be denser than in the disk of the Galaxy. This question has been studied in number of works (Stark \& Bania 1986; Güsten 1989; Stark et al. 1989, 2004), For exemple, Stark et al. (2004) have found that the minimum density (in $\mathrm{cm}^{-3}$ ) for a cloud to withstand the tides can be expressed as:

$n \approx 10^{3.5} \mathrm{~cm}^{-3}\left[\frac{\kappa}{1000 \mathrm{Gyr}^{-1}}\right]^{2}$

where $\kappa$ is the epicyclic frequency, which can be estimated to $1036 \mathrm{Gyr}^{-1}$ for a radius of $150 \mathrm{pc}$ and to $506 \mathrm{Gyr}^{-1}$ for a radius of $450 \mathrm{pc}$ (Stark et al. 2004). Assuming a sun-GC distance of $8.5 \mathrm{kpc}, 150 \mathrm{pc}$ is the radius of the GCR. Therefore, the critical density is $\sim 3 \times 10^{3} \mathrm{~cm}^{-3}$. The clouds with non-circular velocities are located at larger galactocentric radius than the GCR, therefore the critical density is lower. For instance, the critical density for a cloud located at $450 \mathrm{pc}$ is a factor of $\sim 4$ lower 


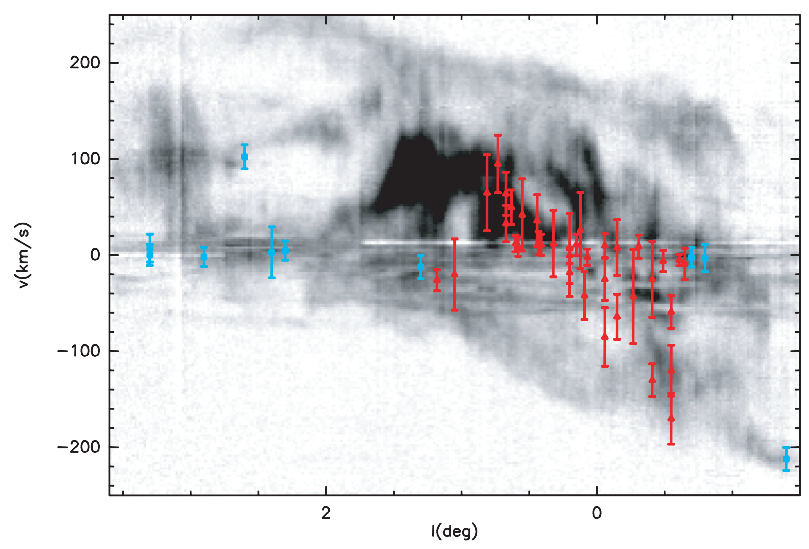

Fig. 5. In grey-levels we show the longitude-velocity diagram obtained integrating the $\mathrm{CO}(1-0)$ data by Bally et al. (1987) in the latitude range $-0.3^{\circ}<b<0.3^{\circ}$. Red triangles and bars represent the peak velocity and the full width at half maximum (FWHM) of the radio recombination lines detected in the longitude range $1.18^{\circ}>l>-0.55^{\circ}$ (Pauls \& Mezger 1975). Blue squares and bars represent the peak velocity and the FWHM of the radio recombination lines detected at $l>1.18^{\circ}$ and $l<-0.55^{\circ}$ (Paladini et al. 2003).

than for a cloud located at $150 \mathrm{pc}$ (Stark et al. 2004). Therefore, the $\mathrm{CS}$ and $\mathrm{SiO}$ clouds in non-circular orbits are gravitationally bound and the dust lanes do not contain diffuse gas only (see also Stark et al. 2004).

However, there are no signs of star formation in the dust lanes. The surveys of hydrogen recombination lines (at centimeter wavelengths) in the inner 2 degrees (Pauls \& Mezger 1975) show that the HII regions are mostly concentrated along a line that runs through the origin of the $l v$ plane indicating that the star formation is confined to the GCR (see Fig. 5). We have also looked for HII regions at larger longitudes in the catalog by Paladini et al. (2003). Figure 5 shows that there are two HII regions at longitude $l \sim-0.6^{\circ}$ associated with the structure $\mathrm{P}$ (or to the spiral arms in the line of sight) and one ionized region at $l=-1.4^{\circ}$ associated with $\mathrm{Sgr} \mathrm{E}$ (and therefore to the GCR). At longitudes $l>1.2^{\circ}$ all the known HII regions are most likely associated with the spiral arms except one source with $l=2.6^{\circ}$ and $v=102 \mathrm{~km} \mathrm{~s}^{-1}$ that could be associated with the structure J. However this structure is basically seen at latitudes $b<-0.1^{\circ}$ (see Fig. 4) and the HII region is located at a latitude $b=0.1^{\circ}$ (Paladini et al. 2003). Therefore, the data available show no signs of star formation in the cloud complexes associated to the dust lanes (Clump 2, J, K, M). However, we can not rule out the presence of ultracompact HII regions (UC-HII) embedded in the dense clumps since very young HII regions could be optically thick at centimeter wavelengths. The presence of isolated UC-HII regions is a possibility that can only be ruled out by deep high resolution searches for recombination line emission at millimeter wavelengths. Nevertheless, such very young UC-HII regions are usually associated with more evolved HII regions that should have been detected by Pauls \& Mezger (1975) and the surveys compiled by Paladini et al. (2003). In summary, with the available recombination line data data there are no signs of massive star formation in the dust lanes.

Indeed, the lack of star formation along the dust lanes is common in the observations of early-type barred galaxies, where there is star formation in the nuclear ring and at the extremities of the bar, but there are no signs of star formation in the dust lanes along the bar (see for instance Tubbs 1982; Reynaud \& Downes 1998; Sheth et al. 2000). The inhibition of the star formation can be an effect of the high velocities, relative to the gas in the dust lanes, of the dense clouds whose orbits intersect the dust lanes (Tubbs 1982). These velocities are much higher in barred than in normal galaxies. The models by Tubbs (1982) showed that the clouds that enter the dust lanes at velocities higher than a critical value ranging between 20 and $60 \mathrm{~km} \mathrm{~s}^{-1}$ must be inhibited from forming stars to explain the observations of NGC 5383. The high velocity can cause a quick compression of the clouds followed by a rapid expansion which disperses the majority of the cloud. This mechanism has also been invoked by Reynaud \& Downes (1998) to explain the observations of NGC 1530. This mechanism could also inhibit the star formation in the gas with non-circular velocities in the GC.

\section{SiO abundances and the origin of the SiO emission}

As already mentioned, the $\mathrm{SiO} / \mathrm{CS}$ line ratio is expected to depend mainly on the relative abundance of the two molecules. For example, in the cloud sample studied by Hüttemeister (1993) and Hüttemeister et al. (1998), which is basically composed by clouds of the GCR and the $l=1.3^{\circ}$-complex, the SiO abundance shows a clear trend to increase from $2 \times 10^{-10}$ to $10^{-8}$ with increasing $\mathrm{SiO}(2-1) / \mathrm{CS}(2-1)$ line ratios in the range $0.1-0.6$. These should also be the $\mathrm{SiO}$ abundances of the sources of this paper with detected $\mathrm{SiO}$.

We have derived the $\mathrm{SiO}$ abundances for some sources of our sample using the ${ }^{13} \mathrm{CO}$ data and LVG calculations. Table 1 shows the $\mathrm{SiO}$ column densities obtained with the typical temperature $\left(T_{\mathrm{K}}=20 \mathrm{~K}\right)$ and density $\left(n\left(\mathrm{H}_{2}\right) \sim 10^{4.5} \mathrm{~cm}^{-3}\right)$ of the $\mathrm{SiO}$ clouds in the GC (Martín-Pintado et al. 1997; Hüttemeister et al. 1998). The abundance of ${ }^{13} \mathrm{CO}$ relative to $\mathrm{H}_{2}$ is $5 \times 10^{-6}$ in the Galactic center (see Hüttemeister et al. 1998, and references therein). Therefore, the $\mathrm{SiO}$ to ${ }^{13} \mathrm{CO}$ column density ratios imply abundances of $\mathrm{SiO}$ relative to $\mathrm{H}_{2}$ of $(0.5-8) \times 10^{-9}$ for the sources with detected $\mathrm{SiO}$, including those in the structures $\mathrm{K}$ and $\mathrm{M}$. These abundances are in agreement with the results of Hüttemeister et al. (1998) and Martín-Pintado et al. (1997, 2000) for clouds located mainly in the GCR and the $l=1.3^{\circ}$ complex. For one source in the Sgr B2 area $\left(l=0.83^{\circ}, b=\right.$ $-0.18^{\circ}, v=57 \mathrm{~km} \mathrm{~s}^{-1}$ ) and two in the feature $\mathrm{P}$ we have even measured a $\mathrm{SiO}$ abundance of $\sim 10^{-8}$, which is comparable to the highest abundances derived by Hüttemeister et al. (1998) in the $l=1.3^{\circ}$-complex.

The $\mathrm{SiO}$ abundance shows extreme variations in the Galaxy. The abundance is $<5 \times 10^{-12}$ in dark clouds (Ziurys et al. 1989) and $10^{-11}-10^{-10}$ in diffuse translucent clouds and in spiral arms clouds (Greaves et al. 1996; Turner 1998; Lucas \& Liszt 2000). Schilke et al. (2001) have measured an abundance of $\sim 10^{-11}$ in the photo-dissociation region (PDR) of the Orion bar and S140. In contrast, the $\mathrm{SiO}$ abundance in shocked regions associated to star formation is $\sim 10^{-8}$ (Martin-Pintado et al. 1992; Bachiller $\&$ Perez Gutierrez 1997). In these regions the $\mathrm{Si}$ and $\mathrm{SiO}$ abundances in the gas phase are increased by the processing of the dust grains by shock waves (Schilke et al. 1997). Thus, the $\mathrm{SiO}$ abundances in the non-circular velocity GC clouds measured in this work and those derived in previous works for other GC clouds (Martín-Pintado et al. 1997, 2000; Hüttemeister et al. 1998), are close to the values found in gas shocked by molecular outflows from protostars. Measurements of the $\mathrm{SiO}$ abundances in the nuclei of external galaxies like NGC 253, M 82, NGC 1068 or IC 342 (García-Burillo et al. 2000, 2001; Usero et al. 2004, 2005) give values from $10^{-10}$ to a few $10^{-9}$, comparable to those measured in the GC. 
Two main scenarios have been invoked to explain the widespread high abundances of $\mathrm{SiO}$ in the GC and the center of the nearby galaxies cited above:

(i) Grain sputtering by shocks waves of different origins (Martín-Pintado et al. 1997). Hüttemeister et al. (1998) have found evidence of a link between the large scale Galactic dynamics and the origin of the shocks in the GC (high $\mathrm{SiO}$ abundances in the $l=1.3^{\circ}$-complex, which they proposed to be due to the interaction of $x_{1}$ and $x_{2}$ orbits). García-Burillo et al. (2000) and Usero et al. (2005) also use shocks related to the galactic dynamics to explain the $\mathrm{SiO}$ emission in NGC 253 and IC 342.

(ii) More recently, a second mechanism has been proposed for regions in the vicinity of X-ray sources: the destruction of small silicate grains by energetic X-rays. This mechanism could play a role in the GCR (Martín-Pintado et al. 2000) and close to the AGN in NGC 1068 (Usero et al. 2004).

As it is well known, the arena of energetic phenomena is in the inner regions of the GC (birth and death of massive stars, energetic radiation, shocks, ...) making it very complex to interpret the $\mathrm{SiO}$ observations of the GCR clouds. In contrast, as discussed above, there are no signs of star formation in the dust lanes and the $\mathrm{SiO}$ abundances are as high as in the other GC sources. The high $\mathrm{SiO}$ abundances measured in the GC clouds with non-circular velocities can be due to the strong shocks that created the dust lanes. This scenario has also been proposed by Meier \& Turner (2005) to interpret the detection of $\mathrm{HNCO}$ and $\mathrm{CH}_{3} \mathrm{OH}$ emission along the bar of IC 342 . Usero et al. (2005) have also detected $\mathrm{SiO}$ emission in the dust lane along the bar of IC 342 and they have measured high $\mathrm{SiO}$ abundances of a few $10^{-9}$ (about a factor of 5-10 higher than in the nuclear ring). They interpret their findings as the effect of largescale shocks driven by cloud-cloud collisions along the potential well of the bar.

\section{Conclusions}

We have shown the presence of dense gas in all the kinematical components of the Galactic center, including the structures with non-circular velocities like the dust lanes along the bar. The gas density of these clouds are higher than the critical density for cloud stability. However, in the GC there are no signs of star formation except in the nuclear ring (the GCR). The high relative velocities and shear expected in other kinematical structures, in particular in those associated with the dust lanes, could inhibit the star formation. This is the mechanism proposed to explain the lack of star formation in the dust lanes along the bar of Galaxies like NGC 5383 and NGC 1530. The high measured abundances of one of the most representative molecules of the GC shock chemistry ( $\mathrm{SiO}$ ) in the cloud components associated with the dust lanes and elongated orbits supports this scenario. Furthermore, it gives us important hints on the origin of the emission of this molecule. The origin of the shocks and therefore the GC molecular chemistry can indeed be strongly influenced by the global dynamics of the Galaxy. More observations of the molecular chemistry in face-on barred galaxies would be very interesting for a better understanding of the links between gas dynamics and molecular chemistry.

Acknowledgements. We thank Jonathan Braine for a critical reading of the manuscript and Sergio Martin for helping with the $30 \mathrm{~m}$ observations. NJR-F has been partially supported by a Marie Curie Fellowship of the European Community program "Improving Human Research Potential and the Socioeconomic Knowledge base" under contract number HPMF-CT-2002-01677. J.M.-P. Acknowledges support by the Spanish Ministerio de Educación y Ciencia under projects AYA 2002-10113-E, AYA 2003-02785-E, and ESP 2004-00665.

\section{References}

Athanassoula, E. 1992, MNRAS, 259, 345

Bachiller, R., \& Perez Gutierrez, M. 1997, ApJ, 487, L93

Bally, J., Stark, A. A., Wilson, R. W., \& Henkel, C. 1987, ApJS, 65, 13

Bally, J., Stark, A. A., Wilson, R. W., \& Henkel, C. 1988, ApJ, 324, 223

Bania, T. M. 1977, ApJ, 216, 381

Binney, J., Gerhard, O. E., Stark, A. A., Bally, J., \& Uchida, K. I. 1991, MNRAS, 252, 210

Bitran, M., Alvarez, H., Bronfman, L., May, J., \& Thaddeus, P. 1997, A\&AS, 125,99

Contopoulos, G., \& Papayannopoulos, T. 1980, A\&A, 92, 33

Dahmen, G., Huettemeister, S., Wilson, T. L., et al. 1997, A\&AS, 126, 197

Fux, R. 1999, A\&A, 345, 787

García-Burillo, S., Martín-Pintado, J., Fuente, A., \& Neri, R. 2000, A\&A, 355, 499

García-Burillo, S., Martín-Pintado, J., Fuente, A., \& Neri, R. 2001, ApJ, 563, L27

Greaves, J. S., Ohishi, M., \& Nyman, L.-A. 1996, A\&A, 307, 898

Güsten, R. 1989, in The Center of the Galaxy, ed. M. Morris, IAU Symp., 136, 89

Hüttemeister, S. 1993, Ph.D. Thesis

Hüttemeister, S., Dahmen, G., Mauersberger, R., et al. 1998, A\&A, 334, 646

Hüttemeister, S., Wilson, T. L., Bania, T. M., \& Martin-Pintado, J. 1993, A\&A, 280,255

Jackson, J. M., Heyer, M. H., Paglione, T. A. D., \& Bolatto, A. D. 1996, ApJ, 456, L91

Linke, R. A., \& Goldsmith, P. F. 1980, ApJ, 235, 437

Lis, D. C., Serabyn, E., Zylka, R., \& Li, Y. 2001, ApJ, 550, 761

Liszt, H. S., \& Burton, W. B. 1978, ApJ, 226, 790

Lucas, R., \& Liszt, H. S. 2000, A\&A, 355, 327

Martin, C. L., Walsh, W. M., Xiao, K., et al. 2004, ApJS, 150, 239

Martin-Pintado, J., Bachiller, R., \& Fuente, A. 1992, A\&A, 254, 315

Martín-Pintado, J., de Vicente, P., Fuente, A., \& Planesas, P. 1997, ApJ, 482, L45

Martín-Pintado, J., Gaume, R. A., Rodríguez-Fernández, N., de Vicente, P., \& Wilson, T. L. 1999, ApJ, 519, 667

Martín-Pintado, J., de Vicente, P., Rodríguez-Fernández, N. J., Fuente, A., \& Planesas, P. 2000, A\&A, 356, L5

Martín-Pintado, J., Rizzo, J. R., de Vicente, P., Rodríguez-Fernández, N. J., \& Fuente, A. 2001, ApJ, 548, L65

Meier, D. S., \& Turner, J. L. 2005, ApJ, 618, 259

Oka, T., Hasegawa, T., Handa, T., Hayashi, M., \& Sakamoto, S. 1996, ApJ, 460, 334

Oka, T., Hasegawa, T., Hayashi, M., Handa, T., \& Sakamoto, S. 1998a, ApJ, 493, 730

Oka, T., Hasegawa, T., Sato, F., Tsuboi, M., \& Miyazaki, A. 1998b, ApJS, 118, 455

Paladini, R., Burigana, C., Davies, R. D., et al. 2003, A\&A, 397, 213

Pauls, T., \& Mezger, P. G. 1975, A\&A, 44, 259

Reynaud, D., \& Downes, D. 1998, A\&A, 337, 671

Rodríguez-Fernández, N. J., Martín-Pintado, J., de Vicente, P., et al. 2000, A\&A, 356,695

Rodríguez-Fernández, N. J., Martín-Pintado, J., Fuente, A., et al. 2001, A\&A, 365,174

Rodríguez-Fernández, N. J., Martín-Pintado, J., Fuente, A., \& Wilson, T. L. 2004, A\&A, 427, 217

Sato, F., Hasegawa, T., Whiteoak, J. B., \& Miyawaki, R. 2000, ApJ, 535, 857

Sawada, T., Hasegawa, T., Handa, T., et al. 2001, ApJS, 136, 189

Schilke, P., Walmsley, C. M., Pineau des Forets, G., \& Flower, D. R. 1997, A\&A, 321, 293

Schilke, P., Pineau des Forêts, G., Walmsley, C. M., \& Martín-Pintado, J. 2001, A\&A, 372, 291

Scoville, N. Z. 1972, ApJ, 175, L127

Sheth, K., Regan, M. W., Vogel, S. N., \& Teuben, P. J. 2000, ApJ, 532, 221

Sofue, Y. 1995, PASJ, 47, 551

Stark, A. A., Bally, J., Wilson, R. W., \& Pound, M. W. 1989, in The Center of the Galaxy, IAU Symp., 136, 129

Stark, A. A., \& Bania, T. M. 1986, ApJ, 306, L17

Stark, A. A., Martin, C. L., Walsh, W. M., et al. 2004, ApJ, 614, L41

Tsuboi, M., Handa, T., \& Ukita, N. 1999, ApJS, 120, 1

Tubbs, A. D. 1982, ApJ, 255, 458

Turner, B. E. 1998, ApJ, 495, 804

Usero, A., García-Burillo, S., Fuente, A., Martín-Pintado, J., \& Rodríguez-Fernández, N. J. 2004, A\&A, 419, 897

Usero, A., Garcia-Burillo, S., Martin-Pintado, J., Fuente, A., \& Neri, R. 2005, ArXiv Astrophysics e-prints

Wilson, T. L., Ruf, K., Walmsley, C. M., et al. 1982, A\&A, 115, 185

Ziurys, L. M., Friberg, P., \& Irvine, W. M. 1989, ApJ, 343, 201 


\section{Online Material}


N. J. Rodriguez-Fernandez et al.: Dynamics and molecular chemistry in the Galactic center, Online Material p 2

Table 2. Galactic longitude and latitude (in degrees), velocity $\left(\mathrm{km} \mathrm{s}^{-1}\right)$, widths $\left(\mathrm{km} \mathrm{s}^{-1}\right)$, and intensities $(\mathrm{K})$ of the CS lines. In addition, the $\mathrm{SiO}$ to CS intensity ratio is also shown. The numbers in parentheses are the uncertainties on the last digit. The positions observed with the IRAM $30 \mathrm{~m}$ telescope are indicated with a superscript. All the other positions have been observed with the $12 \mathrm{~m}$ telescope.

\begin{tabular}{|c|c|c|c|c|c|}
\hline$l$ & $b$ & $v$ & $\Delta v$ & $I(\mathrm{CS})$ & $\frac{I(\mathrm{SiO})}{I(\mathrm{CS})}$ \\
\hline \multicolumn{6}{|c|}{$l=1.3$-complex region } \\
\hline$+1.403^{a}$ & -0.150 & $29(1)$ & $22(1)$ & $0.58(3)$ & $0.19(2)$ \\
\hline$+1.310^{a}$ & -0.130 & $33(1)$ & $23(1)$ & $1.58(9)$ & $0.33(3)$ \\
\hline$+1.403^{a}$ & -0.150 & $123(2)$ & $16(1)$ & $0.36(3)$ & $0.17(3)$ \\
\hline$+1.403^{a}$ & -0.150 & $72(1)$ & $33(1)$ & $0.49(3)$ & $0.33(3)$ \\
\hline$+1.310^{a}$ & -0.130 & $85(1)$ & $32(1)$ & $1.53(9)$ & $0.33(3)$ \\
\hline$+1.310^{a}$ & -0.130 & $135(1)$ & $11(4)$ & $0.16(9)$ & $\leq 0.42$ \\
\hline+1.218 & +0.100 & 119(4) & $56(7)$ & $0.39(3)$ & $0.63(8)$ \\
\hline+1.218 & +0.100 & 119(2) & $17(1)$ & $0.91(3)$ & $0.24(3)$ \\
\hline+1.218 & +0.100 & $163(3)$ & $28(4)$ & $0.26(3)$ & $0.34(11)$ \\
\hline$+1.460^{a}$ & +0.270 & $78(1)$ & $32(5)$ & $1.43(7)$ & $0.28(2)$ \\
\hline$+1.460^{a}$ & +0.270 & $116(2)$ & $16(1)$ & $0.64(7)$ & $0.25(6)$ \\
\hline$+1.698^{a}$ & -0.150 & $3(3)$ & $15(3)$ & $0.09(3)$ & $0.7(3)$ \\
\hline$+1.522^{a}$ & -0.150 & $11(2)$ & $24(1)$ & $0.41(4)$ & $0.27(4)$ \\
\hline+1.560 & -0.300 & $-26(1)$ & $29(1)$ & $0.54(4)$ & $0.46(6)$ \\
\hline$+1.522^{a}$ & -0.150 & $-23(2)$ & $23(1)$ & $0.22(4)$ & $0.18(6)$ \\
\hline$+1.403^{a}$ & -0.150 & $-41(1)$ & $8(1)$ & $0.15(3)$ & $\leq 0.11$ \\
\hline$+1.403^{a}$ & -0.150 & $-22(2)$ & $15(1)$ & $0.30(3)$ & $0.20(4)$ \\
\hline$+1.310^{a}$ & -0.130 & $-13(2)$ & $23(1)$ & $0.73(9)$ & $0.25(7)$ \\
\hline+1.620 & -0.050 & $152(5)$ & $30(8)$ & $0.10(6)$ & $0.6(6)$ \\
\hline+1.708 & -0.150 & $40(2)$ & $25(2)$ & $0.20(2)$ & $0.57(9)$ \\
\hline$+1.698^{a}$ & -0.150 & $42(3)$ & $19(3)$ & $0.39(3)$ & $0.31(4)$ \\
\hline$+1.522^{a}$ & -0.150 & $56(2)$ & $26(1)$ & $0.27(4)$ & $0.22(5)$ \\
\hline+1.620 & -0.050 & $55(5)$ & $16(1)$ & $0.54(5)$ & $0.57(7)$ \\
\hline \multicolumn{6}{|c|}{ Clump 2} \\
\hline+3.329 & +0.425 & $30(1)$ & $33(1)$ & $1.22(6)$ & $0.34(3)$ \\
\hline+3.329 & +0.425 & 150(1) & $32(4)$ & $0.24(6)$ & $0.3(2)$ \\
\hline+3.235 & +0.425 & $68(3)$ & $22(3)$ & $0.59(6)$ & $0.47(7)$ \\
\hline+3.235 & +0.425 & $26(3)$ & $31(3)$ & $0.63(6)$ & $0.32(5)$ \\
\hline+3.219 & +0.250 & $84(1)$ & $34(3)$ & $0.38(6)$ & $0.30(10)$ \\
\hline+3.219 & +0.250 & 152(1) & $58(4)$ & $0.33(6)$ & $0.47(13)$ \\
\hline+3.210 & +0.263 & $77(1)$ & $27(2)$ & $0.45(6)$ & $0.32(8)$ \\
\hline+3.210 & +0.263 & 146(1) & $55(3)$ & $0.33(6)$ & $0.29(11)$ \\
\hline+3.193 & +0.100 & 101(1) & $27(2)$ & $0.78(6)$ & $0.35(7)$ \\
\hline+3.193 & +0.100 & 142(1) & $27(3)$ & $0.46(6)$ & $0.22(11)$ \\
\hline+3.172 & +0.175 & 119(1) & $45(1)$ & $0.72(5)$ & $0.44(7)$ \\
\hline+3.172 & +0.175 & 183(2) & $31(6)$ & $0.16(5)$ & $0.9(4)$ \\
\hline+3.171 & +0.300 & $29(1)$ & $29(2)$ & $0.33(5)$ & $0.41(12)$ \\
\hline+3.171 & +0.300 & $84(2)$ & $33(1)$ & $0.92(5)$ & $0.32(4)$ \\
\hline+3.171 & +0.300 & 143(1) & $37(4)$ & $0.19(5)$ & $0.3(2)$ \\
\hline+3.138 & +0.425 & $7(1)$ & $23(2)$ & $0.54(4)$ & $0.32(5)$ \\
\hline+3.138 & +0.425 & $88(2)$ & $28(1)$ & $1.03(4)$ & $0.34(3)$ \\
\hline+3.138 & +0.425 & 130(1) & $30(2)$ & $0.46(4)$ & $0.27(6)$ \\
\hline+3.136 & +0.250 & 131(4) & $32(7)$ & $0.11(5)$ & $0.4(4)$ \\
\hline+3.136 & +0.250 & $88(1)$ & $30(3)$ & $0.35(5)$ & $0.29(12)$ \\
\hline+3.136 & +0.250 & $31(2)$ & $29(1)$ & $0.53(5)$ & $0.47(9)$ \\
\hline+3.074 & +0.375 & $18(2)$ & $37(1)$ & $0.52(6)$ & $0.38(8)$ \\
\hline+3.047 & -0.013 & $59(1)$ & $34(2)$ & $0.35(5)$ & $0.4(2)$ \\
\hline+3.020 & +0.150 & $156(1)$ & $46(1)$ & $0.67(6)$ & $0.45(7)$ \\
\hline+3.020 & +0.050 & 136(1) & $17(1)$ & $0.62(4)$ & $0.36(6)$ \\
\hline+2.895 & +0.050 & 116(1) & $56(3)$ & $0.74(2)$ & $0.39(2)$ \\
\hline
\end{tabular}

Table 2. continued.

\begin{tabular}{|c|c|c|c|c|c|}
\hline$l$ & $b$ & $v$ & $\Delta v$ & $\overline{I I(C S)}$ & $\frac{I(\mathrm{SiO})}{I(\mathrm{CS})}$ \\
\hline \multicolumn{6}{|c|}{ Galactic center ring } \\
\hline \multicolumn{6}{|l|}{ Sgr E } \\
\hline-1.178 & +0.050 & $-182(2)$ & $21(8)$ & $0.15(2)$ & $\leq 0.87$ \\
\hline-1.178 & +0.050 & $-203(2)$ & $14(3)$ & $0.16(2)$ & $\leq 0.87$ \\
\hline \multicolumn{6}{|l|}{ Sgr B } \\
\hline$+0.830^{a}$ & -0.180 & $31(2)$ & $32(3)$ & $1.12(7)$ & $0.29(5)$ \\
\hline$+0.830^{a}$ & -0.180 & $56(1)$ & $27(1)$ & $1.71(7)$ & $0.32(3)$ \\
\hline \multicolumn{6}{|l|}{ Sgr A } \\
\hline+0.227 & -0.050 & $74(2)$ & $21(1)$ & $0.97(5)$ & $0.21(2)$ \\
\hline$+0.150^{a}$ & -0.050 & $25(2)$ & $13(1)$ & $0.52(6)$ & $\leq 0.19$ \\
\hline$+0.150^{a}$ & -0.050 & $61(2)$ & $23(2)$ & $1.30(6)$ & $0.08(1)$ \\
\hline$+0.145^{a}$ & -0.080 & $52(1)$ & $36(2)$ & $2.21(9)$ & $0.20(2)$ \\
\hline$+0.145^{a}$ & -0.150 & $28(1)$ & $22(1)$ & $0.59(3)$ & $0.12(4)$ \\
\hline$+0.145^{a}$ & -0.120 & $32(2)$ & $8(1)$ & $0.44(7)$ & $0.30(7)$ \\
\hline$+0.145^{a}$ & -0.120 & $50(1)$ & $29(1)$ & $0.97(7)$ & $0.25(3)$ \\
\hline$+0.100^{a}$ & -0.120 & $30(3)$ & $25(3)$ & $2.53(7)$ & $0.15(1)$ \\
\hline$+0.100^{a}$ & -0.120 & $68(3)$ & $15(3)$ & $0.85(7)$ & $\leq 0.16$ \\
\hline \multicolumn{6}{|c|}{ Other sources } \\
\hline$+0.830^{a}$ & -0.180 & $99(1)$ & $17(1)$ & $0.67(7)$ & $\leq 0.20$ \\
\hline+0.945 & -0.150 & $73(2)$ & $17(1)$ & $1.18(6)$ & $0.21(2)$ \\
\hline$+0.945^{a}$ & -0.050 & $86(2)$ & $23(2)$ & $1.31(8)$ & $0.28(2)$ \\
\hline$+0.410^{a}$ & +0.030 & $17(3)$ & $20(3)$ & $1.84(4)$ & $0.41(2)$ \\
\hline$+0.410^{a}$ & +0.030 & $37(3)$ & $13(3)$ & $1.38(4)$ & $\leq 0.10$ \\
\hline$+0.410^{a}$ & +0.030 & $-51(3)$ & $19(3)$ & $0.52(4)$ & $0.35(7)$ \\
\hline$+0.410^{a}$ & +0.030 & $-21(3)$ & $21(3)$ & $0.26(4)$ & $0.3(2)$ \\
\hline+0.401 & -0.150 & $12(1)$ & $35(3)$ & $0.24(4)$ & $\leq 0.22$ \\
\hline$+0.390^{a}$ & -0.150 & $-3(1)$ & $9(2)$ & $0.42(2)$ & $\leq 0.11$ \\
\hline$+0.390^{a}$ & -0.150 & $13(1)$ & $28(4)$ & $0.30(2)$ & $\leq 0.15$ \\
\hline+0.227 & -0.050 & $29(1)$ & $18(2)$ & $1.51(5)$ & $0.10(1)$ \\
\hline+0.227 & -0.050 & $-30(2)$ & $28(4)$ & $0.14(5)$ & $\leq 0.51$ \\
\hline$+0.150^{a}$ & -0.050 & $-92(2)$ & $13(2)$ & $0.27(6)$ & $\leq 0.33$ \\
\hline$+0.150^{a}$ & -0.050 & $-65(1)$ & $14(3)$ & $0.14(6)$ & $\leq 0.54$ \\
\hline$+0.145^{a}$ & -0.080 & $-14(1)$ & $10(1)$ & $1.68(9)$ & $\leq 0.08$ \\
\hline$+0.100^{a}$ & -0.120 & $-2(3)$ & $14(3)$ & $0.35(7)$ & $\leq 0.35$ \\
\hline-0.198 & -0.050 & $27(2)$ & $27(1)$ & $0.63(5)$ & $0.11(3)$ \\
\hline-0.198 & -0.050 & $85(3)$ & $33(8)$ & $0.10(5)$ & $0.5(3)$ \\
\hline-0.198 & -0.050 & $-18(1)$ & $21(2)$ & $0.35(5)$ & $\leq 0.17$ \\
\hline-0.460 & -0.300 & $45(4)$ & $34(9)$ & $0.18(4)$ & $\leq 0.25$ \\
\hline-0.460 & -0.300 & $72(2)$ & $18(4)$ & $0.18(4)$ & $\leq 0.25$ \\
\hline$-0.503^{a}$ & -0.050 & $-53(1)$ & $21(2)$ & $0.16(4)$ & $0.9(5)$ \\
\hline$-0.503^{a}$ & -0.050 & $-147(1)$ & $8(3)$ & $0.17(4)$ & $0.6(5)$ \\
\hline$-0.503^{a}$ & -0.050 & $-103(1)$ & $11(2)$ & $1.42(4)$ & $0.18(5)$ \\
\hline$-0.590^{a}$ & -0.050 & $-148(2)$ & $25(6)$ & $0.12(2)$ & $\leq 1.24$ \\
\hline$-0.590^{a}$ & -0.050 & $-116(2)$ & $14(1)$ & $1.02(2)$ & $0.28(1)$ \\
\hline$-0.688^{a}$ & -0.050 & $-129(1)$ & $17(2)$ & $0.31(4)$ & $\leq 0.61$ \\
\hline-0.754 & -0.050 & $-132(2)$ & $61(4)$ & $0.17(4)$ & $\leq 0.25$ \\
\hline \multicolumn{6}{|l|}{$\mathrm{F}$} \\
\hline-0.655 & +0.100 & $-165(2)$ & $20(4)$ & $0.14(3)$ & $\leq 0.24$ \\
\hline-0.655 & +0.100 & $-117(2)$ & $15(4)$ & $0.10(3)$ & $\leq 0.33$ \\
\hline$-0.503^{a}$ & +0.150 & $-145(6)$ & $21(2)$ & $0.59(5)$ & $\leq 0.17$ \\
\hline$-0.503^{a}$ & +0.150 & $-112(1)$ & $20(3)$ & $0.28(5)$ & $\leq 0.33$ \\
\hline-0.285 & +0.100 & $-87(4)$ & $20(9)$ & $0.07(3)$ & $\leq 0.77$ \\
\hline-0.285 & +0.100 & $-51(4)$ & $22(7)$ & $0.09(2)$ & $\leq 0.72$ \\
\hline
\end{tabular}


N. J. Rodriguez-Fernandez et al.: Dynamics and molecular chemistry in the Galactic center, Online Material p 3

Table 2. continued.

\begin{tabular}{|c|c|c|c|c|c|}
\hline $\bar{l}$ & $\overline{b b}$ & $\bar{v}$ & $\overline{\Delta \Delta v}$ & $\overline{I I(C S)}$ & $\frac{I(\mathrm{SiO})}{I(\mathrm{CS})}$ \\
\hline \multicolumn{6}{|r|}{ (tes) } \\
\hline$-0.503^{a}$ & -0.050 & $144(3)$ & $17(4)$ & $0.07(4)$ & $\leq 1.26$ \\
\hline$-0.590^{a}$ & -0.050 & $132(1)$ & $8(1)$ & $0.12(2)$ & $\leq 1.24$ \\
\hline$-0.590^{a}$ & -0.050 & $152(1)$ & $7(2)$ & $0.10(2)$ & $\leq 1.44$ \\
\hline$-0.688^{a}$ & -0.050 & $142(1)$ & $10(1)$ & $0.30(4)$ & $\leq 0.63$ \\
\hline-0.754 & -0.050 & $118(2)$ & $41(5)$ & $0.16(4)$ & $\leq 0.26$ \\
\hline$-0.503^{a}$ & +0.150 & 171(4) & $52(9)$ & $0.12(4)$ & $\leq 0.63$ \\
\hline-0.655 & +0.100 & $161(6)$ & $20(2)$ & $0.29(3)$ & $0.21(5)$ \\
\hline-0.862 & +0.100 & $152(1)$ & $18(1)$ & $0.54(4)$ & $0.24(2)$ \\
\hline-0.950 & +0.150 & $129(1)$ & $23(2)$ & $0.42(5)$ & $0.40(10)$ \\
\hline-0.982 & +0.100 & $130(2)$ & $18(2)$ & $0.30(3)$ & $0.27(5)$ \\
\hline \multicolumn{6}{|c|}{$\mathbf{O}$} \\
\hline+2.895 & +0.050 & $8(2)$ & 24(4) & $0.30(2)$ & $\leq 0.38$ \\
\hline+2.880 & +0.113 & $9(1)$ & $22(2)$ & $0.32(4)$ & $0.28(1)$ \\
\hline+2.720 & -0.150 & $9(4)$ & $19(6)$ & $0.07(4)$ & $\leq 0.55$ \\
\hline+2.610 & +0.000 & $-9(2)$ & $28(5)$ & $0.14(3)$ & $\leq 0.47$ \\
\hline+2.610 & +0.000 & $-39(3)$ & $16(6)$ & $0.07(3)$ & $\leq 0.78$ \\
\hline+2.568 & +0.150 & $4(2)$ & $23(4)$ & $0.12(3)$ & $\leq 0.60$ \\
\hline+2.121 & -0.150 & $-47(1)$ & $13(3)$ & $0.14(3)$ & $\leq 0.37$ \\
\hline+2.121 & -0.150 & $25(4)$ & $49(8)$ & $0.06(3)$ & $\leq 0.68$ \\
\hline+2.056 & -0.300 & $1(2)$ & $23(2)$ & $0.17(3)$ & $\leq 0.31$ \\
\hline+2.056 & -0.300 & $36(1)$ & $18(3)$ & $0.11(3)$ & $\leq 0.44$ \\
\hline \multicolumn{6}{|c|}{$\overline{\mathbf{P}}$} \\
\hline$-0.590^{a}$ & -0.050 & $-41(1)$ & $14(2)$ & $0.28(2)$ & $\leq 0.59$ \\
\hline$-0.688^{a}$ & -0.050 & $-41(1)$ & $26(3)$ & $0.42(4)$ & $\leq 0.47$ \\
\hline$-0.688^{a}$ & -0.050 & $-17(1)$ & $16(2)$ & $0.57(4)$ & $0.51(7)$ \\
\hline$-0.688^{a}$ & -0.050 & $2(1)$ & $25(2)$ & $0.59(4)$ & $0.47(7)$ \\
\hline-0.754 & -0.050 & $-10(2)$ & $37(3)$ & $0.32(4)$ & $0.15(4)$ \\
\hline-0.829 & -0.300 & $-18(1)$ & $40(2)$ & $0.22(3)$ & $\leq 0.35$ \\
\hline-0.906 & -0.150 & $-2(2)$ & $39(2)$ & $0.40(5)$ & $0.24(4)$ \\
\hline-0.655 & +0.100 & $2(2)$ & $24(7)$ & $0.09(3)$ & $\leq 0.35$ \\
\hline-0.862 & +0.100 & $-1(1)$ & 23(3) & $0.25(4)$ & $0.24(5)$ \\
\hline \multicolumn{6}{|c|}{$\mathbf{J}$} \\
\hline+2.720 & -0.150 & $96(1)$ & $23(3)$ & $0.29(4)$ & $0.45(8)$ \\
\hline+2.056 & -0.300 & $141(2)$ & $23(2)$ & $0.16(3)$ & $\leq 0.33$ \\
\hline$+1.958^{a}$ & -0.100 & $122(3)$ & $27(1)$ & $0.56(6)$ & $0.25(3)$ \\
\hline \multicolumn{6}{|c|}{$\mathbf{K}$} \\
\hline$+1.958^{a}$ & -0.100 & $247(1)$ & $9(3)$ & $0.22(6)$ & $\leq 0.32$ \\
\hline+1.708 & -0.150 & $221(4)$ & $31(6)$ & $0.08(2)$ & $\leq 0.85$ \\
\hline$+1.698^{a}$ & -0.150 & $220(3)$ & $20(3)$ & $0.16(3)$ & $\leq 0.20$ \\
\hline$+1.522^{a}$ & -0.150 & $219(3)$ & $16(1)$ & $0.42(4)$ & $\leq 0.09$ \\
\hline$+1.403^{a}$ & -0.150 & $214(6)$ & $21(5)$ & $0.14(3)$ & $0.14(4)$ \\
\hline$+1.310^{a}$ & -0.130 & $204(2)$ & $19(4)$ & $0.11(9)$ & $\leq 0.53$ \\
\hline+0.401 & -0.150 & $192(3)$ & $28(8)$ & $0.08(4)$ & $\leq 0.49$ \\
\hline$+0.390^{a}$ & -0.150 & $195(2)$ & $8(1)$ & $0.39(2)$ & $\leq 0.12$ \\
\hline$+0.145^{a}$ & -0.150 & $175(2)$ & $7(1)$ & $0.20(3)$ & $\leq 0.38$ \\
\hline$+1.698^{a}$ & -0.150 & $185(3)$ & $12(3)$ & $0.11(3)$ & $0.5(2)$ \\
\hline$+1.698^{a}$ & -0.150 & $163(3)$ & $13(3)$ & $0.12(3)$ & $\leq 0.25$ \\
\hline+1.708 & -0.150 & $157(6)$ & 48(9) & $0.53(2)$ & $\leq 0.01$ \\
\hline+0.227 & -0.050 & $176(1)$ & $10(1)$ & $0.20(5)$ & $\leq 0.39$ \\
\hline$+0.150^{a}$ & -0.050 & $154(1)$ & $4(1)$ & $0.20(6)$ & $\leq 0.42$ \\
\hline$+0.145^{a}$ & -0.080 & $177(2)$ & $12(4)$ & $0.21(9)$ & $\leq 0.47$ \\
\hline
\end{tabular}

Table 2. continued.

\begin{tabular}{llllll}
\hline \hline$l$ & $b$ & $v$ & $\Delta v$ & $I(\mathrm{CS})$ & $\frac{I(\mathrm{SIO})}{I(\mathrm{CS})}$ \\
\hline & \multicolumn{5}{c}{$\mathbf{M}$} \\
+0.401 & -0.150 & $-102(4)$ & $53(8)$ & $0.10(4)$ & $\leq 0.42$ \\
$+0.390^{a}$ & -0.150 & $-92(2)$ & $12(4)$ & $0.06(2)$ & $0.7(3)$ \\
$+0.390^{a}$ & -0.150 & $-71(3)$ & $18(8)$ & $0.05(2)$ & $1.0(5)$ \\
$+0.145^{a}$ & -0.150 & $-131(1)$ & $8(3)$ & $0.09(3)$ & $\leq 0.71$ \\
$+0.145^{a}$ & -0.150 & $-107(5)$ & $64(9)$ & $0.10(3)$ & $\leq 0.66$ \\
+0.063 & -0.300 & $-140(2)$ & $38(4)$ & $0.12(3)$ & $\leq 0.40$ \\
-0.460 & -0.300 & $-118(1)$ & $22(1)$ & $0.60(4)$ & $0.09(2)$ \\
-0.829 & -0.300 & $-123(3)$ & $39(9)$ & $0.10(3)$ & $\leq 0.66$ \\
-0.906 & -0.150 & $-133(1)$ & $22(3)$ & $0.19(5)$ & $\leq 0.27$ \\
-0.906 & -0.150 & $-169(2)$ & $27(8)$ & $0.11(5)$ & $\leq 0.40$ \\
$+0.945^{a}$ & -0.050 & $-81(2)$ & $14(3)$ & $0.23(8)$ & $0.3(2)$ \\
$+0.945^{a}$ & -0.050 & $-65(2)$ & $14(3)$ & $0.22(8)$ & $0.18(9)$ \\
\hline \multicolumn{7}{c}{$\mathbf{N}$} & & & \\
-1.102 & -0.250 & $-44(2)$ & $39(4)$ & $0.21(5)$ & $0.26(11)$ \\
-1.189 & -0.300 & $-4(1)$ & $13(4)$ & $0.19(4)$ & $\leq 0.58$ \\
-1.189 & -0.300 & $-42(1)$ & $41(3)$ & $0.43(4)$ & $0.21(5)$ \\
-1.189 & -0.300 & $-103(3)$ & $15(6)$ & $0.08(4)$ & $\leq 1.08$ \\
-1.211 & -0.150 & $-118(2)$ & $25(2)$ & $0.50(4)$ & $0.26(6)$ \\
-1.211 & -0.150 & $-42(1)$ & $34(2)$ & $0.46(4)$ & $0.28(6)$ \\
\hline
\end{tabular}


N. J. Rodriguez-Fernandez et al.: Dynamics and molecular chemistry in the Galactic center, Online Material p 4

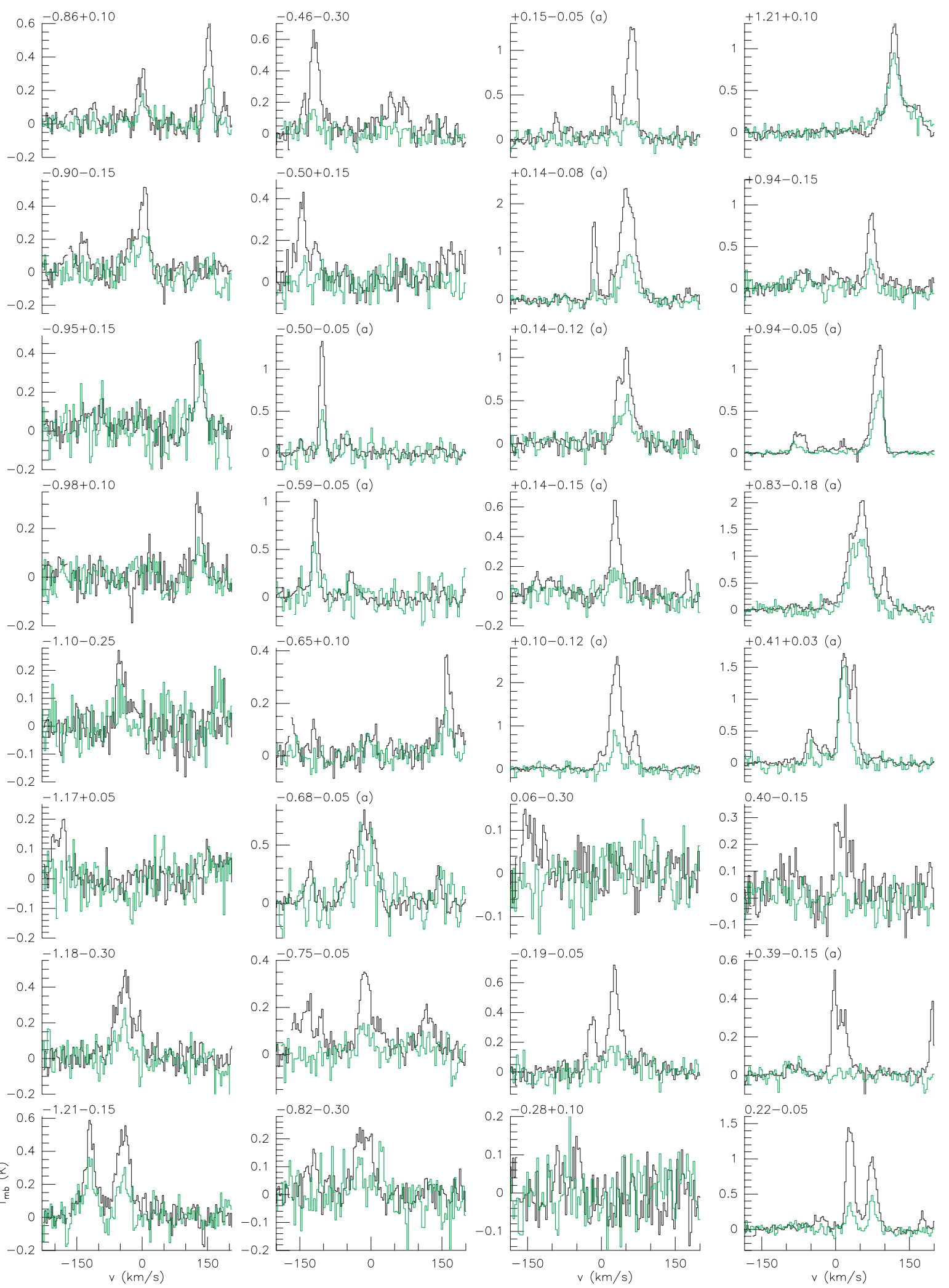

Fig. 3. $\mathrm{CS}(2-1)$ spectra (black lines) and $\mathrm{SiO}(2-1)$ spectra (multiplied by 2 , green lines) towards all the observed positions, which are written in the upper-left corner (in Galactic coordinates). The positions observed with the 30m telescope are indicated with a label (a). The velocity scale is the same for all the spectra in the same column. 
N. J. Rodriguez-Fernandez et al.: Dynamics and molecular chemistry in the Galactic center, Online Material p 5

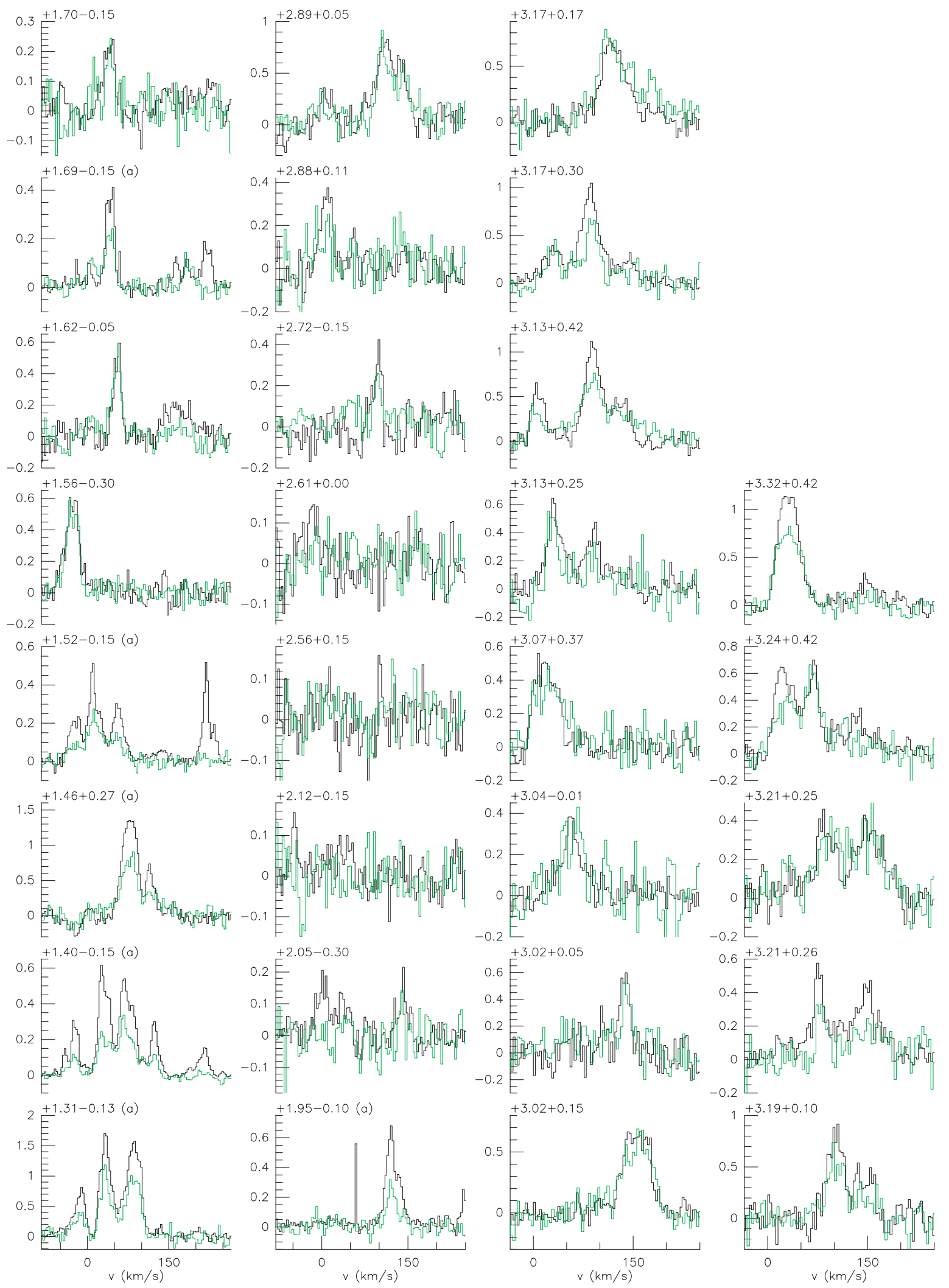

Fig. 3. continued. 